\title{
Effect of helium addition on discharge characteristics in a flat fluorescent lamp
}

Seong-Eui Lee, Ho-Nyeon Lee, and Hyoung-Bin Park

Materials and Devices Research Center, Samsung Advanced Institute of Technology, Suwon 440-600, Korea

Kyo Sung Lee

Department of Electronics Engineering, Sejong University, Seoul 143-747, Korea

Kyung Cheol Choi ${ }^{\text {a) }}$

Department of Electrical Engineering and Computer Science, Korea Advanced Institute of Science and Technology (KAIST), Daejeon 305-701, Korea

(Received 2 February 2005; accepted 29 September 2005; published online 11 November 2005)

\begin{abstract}
The discharge characteristics of a multielectrode dual coplanar in a mercury-free flat fluorescent lamp were investigated using brightness-efficiency measurement and the infrared (IR) spectrum and intensified charge coupled device (ICCD) characteristics. The level of brightness was above $14900 \mathrm{~cd} / \mathrm{m}^{2}$ under the conditions of neon- $-50 \%$ xenon-8\% He gas composition, 150 Torr pressure, and $20 \mathrm{kHz}$ alternating current pulse. The ICCD results revealed a faster and wider discharge with a $\mathrm{Ne}-50 \% \mathrm{Xe}-8 \% \mathrm{He}$ gas composition. The effect of adding helium (He) to $\mathrm{Ne}-50 \%$ $\mathrm{Xe}$ revealed a faster peak emission, as confirmed by ICCD images. From the gated IR emission spectrum, the intensity ratio of $I_{823 \mathrm{~nm}} / I_{828} \mathrm{~nm}$ was $\sim 8 \%$ higher with $\mathrm{Ne}-50 \% \mathrm{Xe}-8 \%$ He than with $\mathrm{Ne}-50 \%$ Xe under the same pressure and applied voltage conditions. (C) 2005 American Institute of Physics. [DOI: 10.1063/1.2127121]
\end{abstract}

\section{INTRODUCTION}

The technology trend for thin-film transistor liquidcrystal displays (TFT-LCDs) is moving away from small-size displays for monitors to large-size displays for digital television application. Thus, much of the current research is focusing on large-size and high-efficiency backlighting. In the case of external electrode fluorescent lamps (EEFLs) and cold cathode fluorescent lamps (CCFLs), the uniformity of the backlight unit is a key problem as the panel size becomes increasingly larger. In addition, environmental issues related to mercury are also a major concern. Therefore, another type of flat lamp is needed for large-size TFT-LCDs. As an alternative, mercury ( $\mathrm{Hg}$ )-free flat fluorescent lamps have already been widely studied as potential pollution-free, high-luminance, long-lifetime liquid-crystal display (LCD)backlights. ${ }^{1,2}$ However, their efficiency and luminance still require improvement for the demands of commercialization. Xenon is widely used as a discharge gas, often in combination with buffer gases, for low reactivity and high efficiency. Ikeda et al. previously developed small-size coplanar flat discharge lamps that can be used in car navigation systems, where the lamp is composed of a pair of coplanar electrodes and operated by alternating-current (ac) pulse driving. ${ }^{1}$ In this case, the system efficiency was around $21 \mathrm{~lm} / \mathrm{W}$ at $10000 \mathrm{~cd} / \mathrm{m}^{2}$ for a $5.2 \mathrm{in}$. Xe discharge lamp. Meanwhile, for larger-size backlights, Ilmer et al. developed a lamp composed of hundreds of microdischarges to prevent constriction of the discharge, and the highest system efficiency was around $28 \mathrm{~lm} / \mathrm{W}$ at $7500 \mathrm{~cd} / \mathrm{m}^{2}{ }^{2}$

In a previous work, we reported the results of a dual-

${ }^{a)}$ Electronic mail: kyungcc@ee.kaist.ac.kr coplanar lamp with better efficiency and brightness than a conventional coplanar lamp. ${ }^{3}$ Based on the previous results, an experimental mercury-free flat lamp based on a multielectrode dual-coplanar fluorescent lamp was developed. The electrode shape was changed, as shown in Fig. 1, in order to observe the discharge characteristics with various pressures and gas compositions; helium (He) gas was imported as a buffer gas. The discharge characteristics were examined by infrared (IR) and intensified charge-coupled device (ICCD) images to verify the effect of the He additive.

\section{EXPERIMENT}

In the previous work, the authors introduced a dualcoplanar mercury-free flat fluorescent lamp and presented experimental results showing that the brightness and efficiency are almost doubled for the dual-coplanar lamp compared to the conventional coplanar lamp. ${ }^{3}$ An efficiency of $35.9 \mathrm{~lm} / \mathrm{W}$ was achieved for 250 Torr of $\mathrm{Ne}-30 \%$ Xe gas mixture by $20 \mathrm{kHz}$ ac pulse driving. Numerical analyses indicated that the improvements in brightness and efficiency

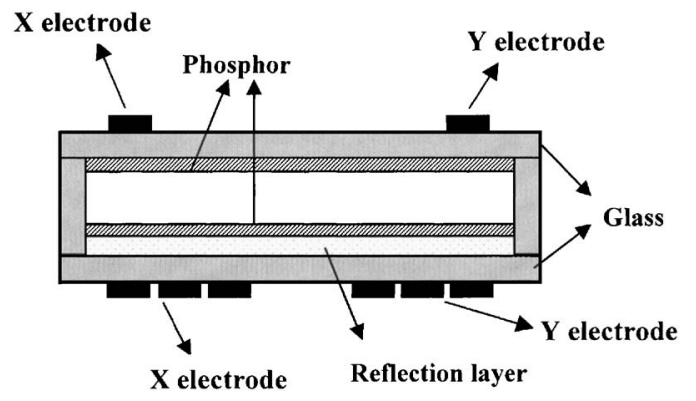

FIG. 1. Schematic diagram of multielectrode dual-coplanar lamp. 
resulted mainly from the less bent electric-field lines in the dual-coplanar lamp. This resulted in fewer electrons near the boundary, less diffusion loss at low pressures and/or low cell-gap ranges, and more effective usage of discharge volume in the dual-coplanar lamps, for which the volume used for discharge is larger and the distance between the produced vacuum ultraviolet (VUV) and phosphor is shorter at high pressures and/or high cell-gap ranges.

Based on this result, the structure shown in Fig. 1 was introduced. The basic concept for this structure is that the discharge starts between the nearest pair of $X$ and $Y$ electrode lines and spreads to the region beyond the electrode lines, resulting in a long electrode gap on average for high efficiency and a short ignition gap for low starting voltages. Mesh electrode lines are used to reduce electrode area and thus the current. The detailed characteristics of the multipleelectrode dual-coplanar lamp discharge and its dependence on various structure parameters, driving condition, materials, and processes will be reported elsewhere soon. In this paper we focus on the discharge gas of the multiple-electrode dualcoplanar lamps, particularly on the effect of helium addition to the previous $\mathrm{Ne}-\mathrm{Xe}$ gas mixture. In the experiment, the electrode lines were formed using a Ag conductive paste on the outer surfaces of the plates, and the line width was $1 \mathrm{~mm}$. The gap between the electrode lines was $40 \mathrm{~mm}$ on the front plate, $12 \mathrm{~mm}$ between the nearest pair of $X$ and $Y$ electrode lines, and $6 \mathrm{~mm}$ between electrode lines of the same polarity on the rear plate. The height of the sealing frame was $4 \mathrm{~mm}$. The front and rear plates were 1.1-mm-thick soda-lime glasses.

The white phosphor used in this experiment was made by mixing red, green, and blue phosphors with a mixing ratio that would yield color coordinates around $(0.31,0.33)$ for the final visible light from the panel. The thickness of the phosphor layer was $0.01 \mathrm{~mm}$ for the front plate and $0.05 \mathrm{~mm}$ for the bottom plate. A $0.02 \mathrm{~mm}$ reflective layer of white dielectric material was formed between the bottom phosphor and the bottom plate. $50 \%$ xenon balanced with Ne buffer gas was used as the standard discharge gas, and the He added gas mixtures were tested and compared with the standard discharge gas. Various pressures and gas mixing ratios were tested. The panel was driven with ac pulses of $20 \mathrm{kHz}$ frequency and $5 \mu$ s pulse width, which were selected after testing various driving conditions. The gated IR emission was obtained with $10 \mathrm{~ns}$ interval using an ICCD spectrometer (C8800-01, Hamamatsu). Discharge images were taken with an ICCD camera (ICCD4710, Andor) at 20 ns gate mode.

\section{RESULTS AND DISCUSSION}

Figure 2 shows the $V_{s}$ and $V_{f}$ characteristics of the flat lamp with different total pressure in $\mathrm{Ne}-50 \% \mathrm{Xe}$ and with the addition of $3 \%$ or $8 \%$ He gas. Here, $V_{f}$ is defined as the ignition voltage of discharge and $V_{s}$ is defined as the discharge-off voltage. The driving condition was square $20 \mathrm{kHz}$ ac pulses with a $5 \mu$ s pulse width. $V_{s}$ and $V_{f}$ linearly increased with a total pressure ranging from 80 to 150 Torr. The addition of He to the Xe lamp caused an increase in $V_{f}$ and $V_{s}$. With a total pressure of 150 Torr, the firing voltage

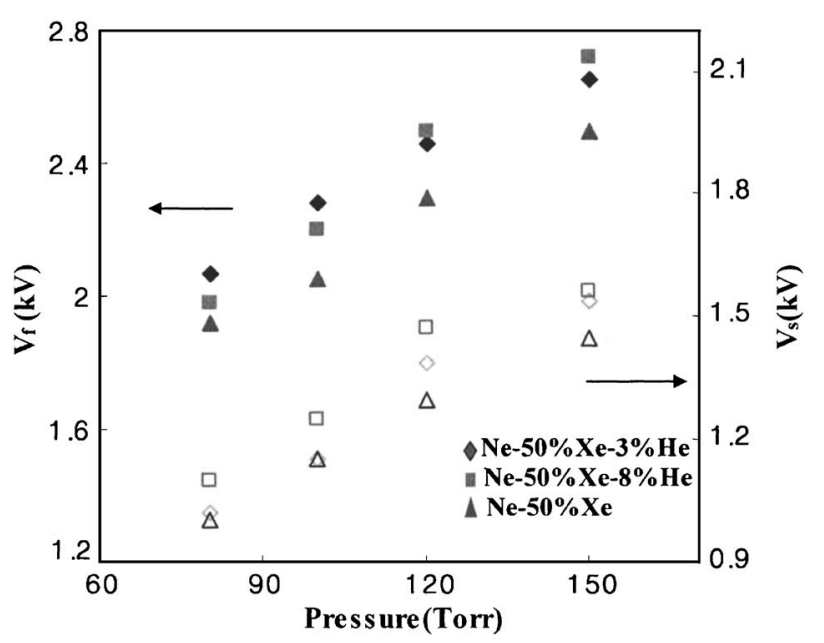

FIG. 2. $V_{s}$ and $V_{f}$ characteristics of multielectrode dual-coplanar lamp with variation in total pressure and different gas compositions.

was $300 \mathrm{~V}$ higher in the $\mathrm{Ne}-50 \% \mathrm{Xe}-8 \%$ He lamp than in the $\mathrm{Ne}-50 \%$ Xe lamp. This difference is contributed to the higher breakdown field in the $\mathrm{Ne}-\mathrm{Xe}-\mathrm{He}$ mixture relative to that in the $\mathrm{Ne}-\mathrm{Xe}$ (Ref. 4) case due to the higher electron momentum transfer cross section for He compared to that for $\mathrm{Ne}$.

The luminance and efficacy characteristics of the dualcoplanar multielectrode lamp are shown in Figs. 3 and 4. Here, luminance and efficacy characteristics are investigated at $V_{s}$. With increasing total pressure, the luminance increased almost linearly. A distinct luminance difference with the addition of $\mathrm{He}$ and a total pressure range of 120-150 Torr were observed. With a total pressure of 150 Torr and a $\mathrm{Ne}-$ $50 \% \mathrm{Xe}-8 \% \mathrm{He}$ gas composition, the luminance was $14900 \mathrm{~cd} / \mathrm{m}^{2}$, more than $40 \%$ higher than the case of $\mathrm{Ne}-$ $50 \% \mathrm{Xe}$. The driving condition was an ac rectangular pulse with $20 \mathrm{kHz}$ frequency and $5 \mu$ s duty cycles. Input power was measured using a power meter. Although the efficiency level was not as sensitive as the brightness change with increasing total pressure, the efficiency was still more than

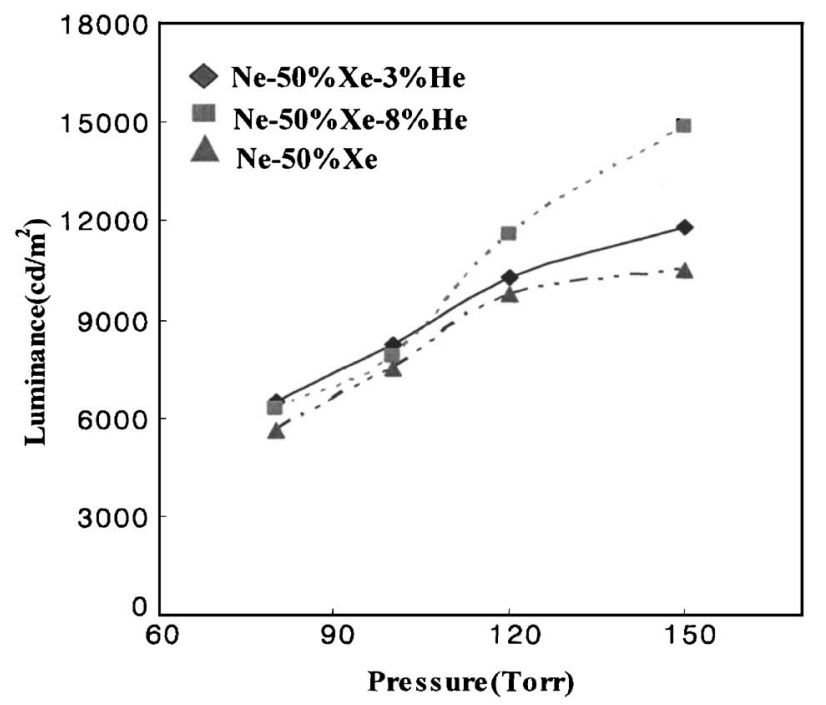

FIG. 3. Luminance of multielectrode dual-coplanar lamp with variation in total pressure and different gas compositions. 


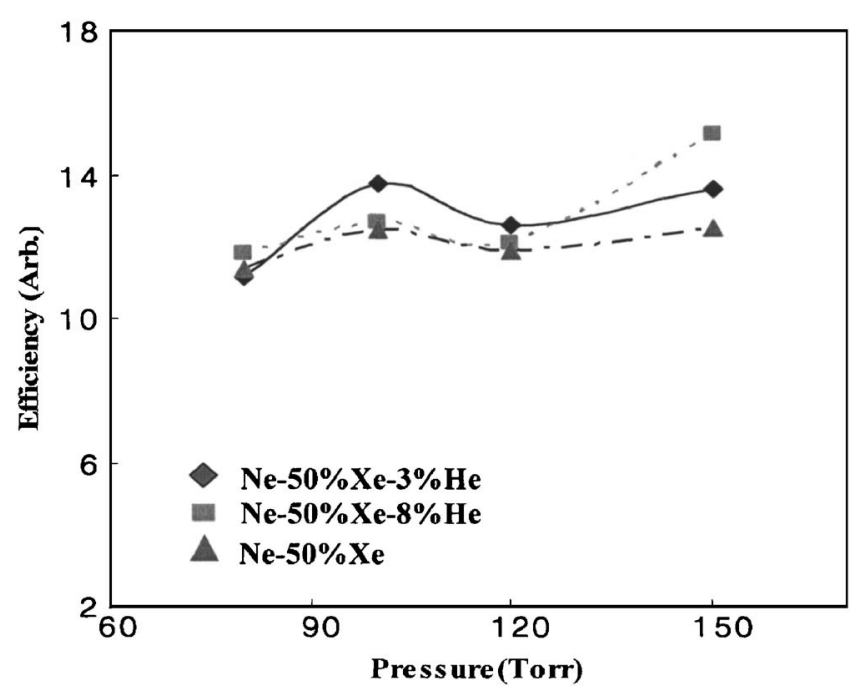

FIG. 4. Efficiency of multielectrode dual-coplanar lamp with variation in total pressure and different gas compositions.

$30 \%$ higher in $\mathrm{Ne}-50 \% \mathrm{Xe}-8 \% \mathrm{He}$ than in $\mathrm{Ne}-50 \% \mathrm{Xe}$ at a total pressure of 150 Torr. In order to access the main factors involved in the brightness and efficiency increment, the IR emission characteristics of $\mathrm{Ne}-50 \% \mathrm{Xe}-8 \% \mathrm{He}$ and $\mathrm{Ne}-50 \%$ Xe were examined at a total pressure of 120 Torr and under
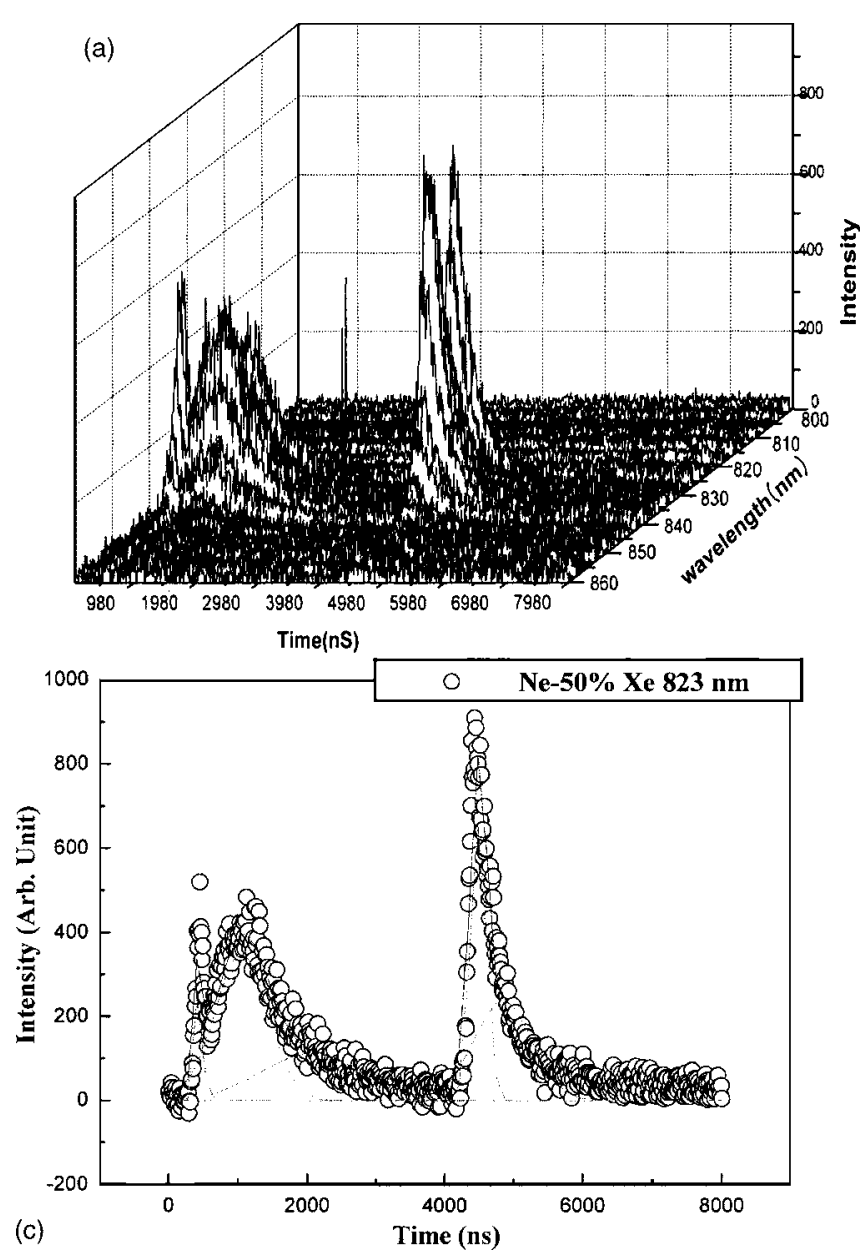

the same previous conditions of $20 \mathrm{kHz}$ frequency and $5 \mu \mathrm{s}$ duty cycles. The IR emission spectrum was obtained at the center position between the $X$ and $Y$ electrodes.

In Fig. 5, various IR emissions are plotted over time for $\mathrm{Ne}-50 \% \mathrm{Xe}-8 \% \mathrm{He}$ and $\mathrm{Ne}-50 \% \mathrm{Xe}$. Both IR spectra show distinct broad peaks related to the pulse duty cycles. The peaks before $4 \mu$ s are from the rising edges of the driving pulse, while those after $5 \mu$ s are from the falling edges of the driving pulse. The curves of $823 \mathrm{~nm}$ in $\mathrm{Ne}-50 \% \mathrm{Xe}$ and $\mathrm{Ne}-50 \% \mathrm{Xe}-8 \% \mathrm{He}$ discharges are deconvoluted, as shown in Figs. 5(c) and 5(d). Each peak for the rising edges and falling edges is first composed of a sharp component followed by broader ones. The sharper components appear to reflect the discharge due to the voltage coming from the external driving pulse and internal wall voltage. For the rising edge, the wall voltage decreases owing to the space charges generated from the discharge following the first discharge. As the driving voltage becomes higher after the first discharge, the total voltage of the induced voltage and wall voltage becomes higher than the firing voltage, thereby explaining the following discharges. 5 For the ensuing discharges, the effective firing voltage is lower than that for the first discharge, as there are enough space charges and metastable originating from the first discharge. ${ }^{6}$ This mechanism can also be applied to the discharges from the following
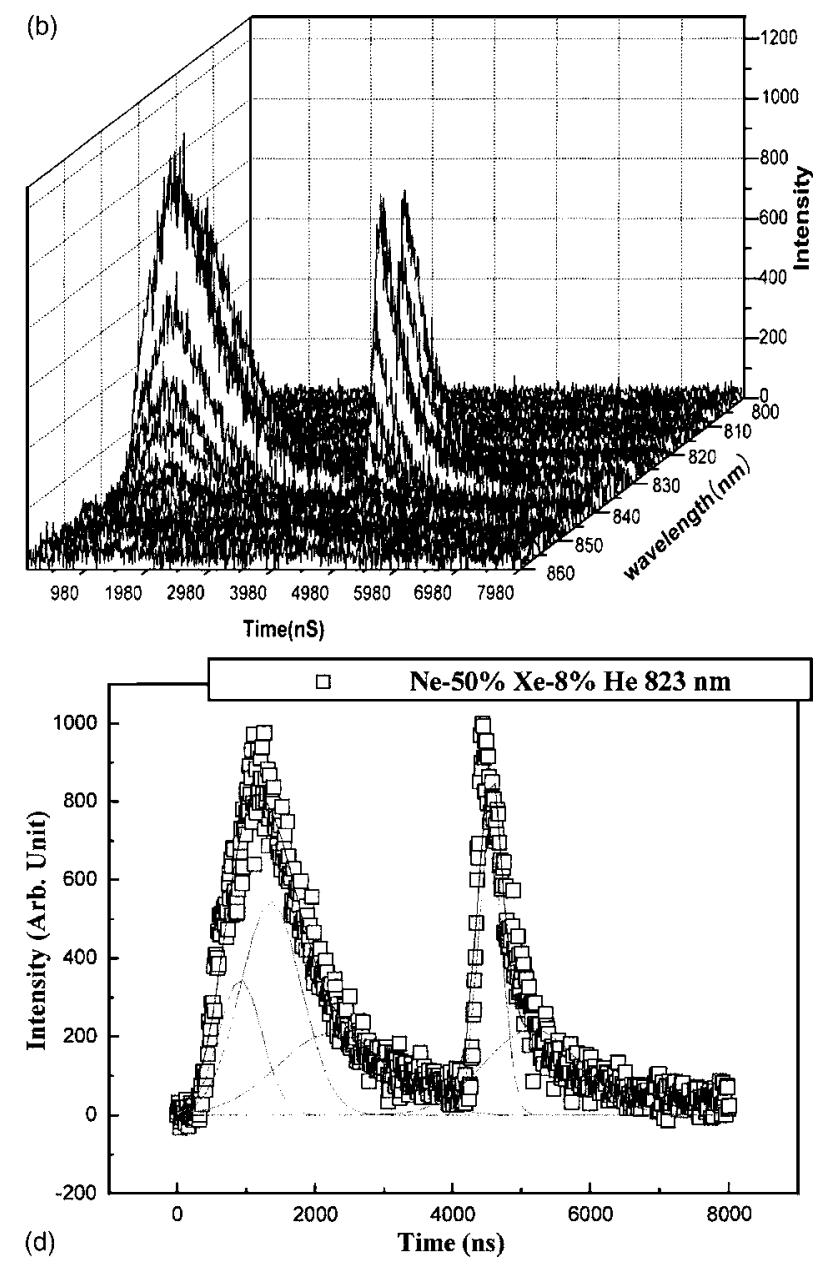

FIG. 5. Variation in infrared emission relative to time and wavelength. (a) Ne-50\% Xe discharge, (b) Ne-50\% Xe-8\% He discharge, (c) deconvoluted curve of $823 \mathrm{~nm}$ in $\mathrm{Ne}-50 \%$ Xe discharge, and (d) deconvoluted curve of $823 \mathrm{~nm}$ in $\mathrm{Ne}-50 \% \mathrm{Xe}-8 \% \mathrm{He}$ discharge. 


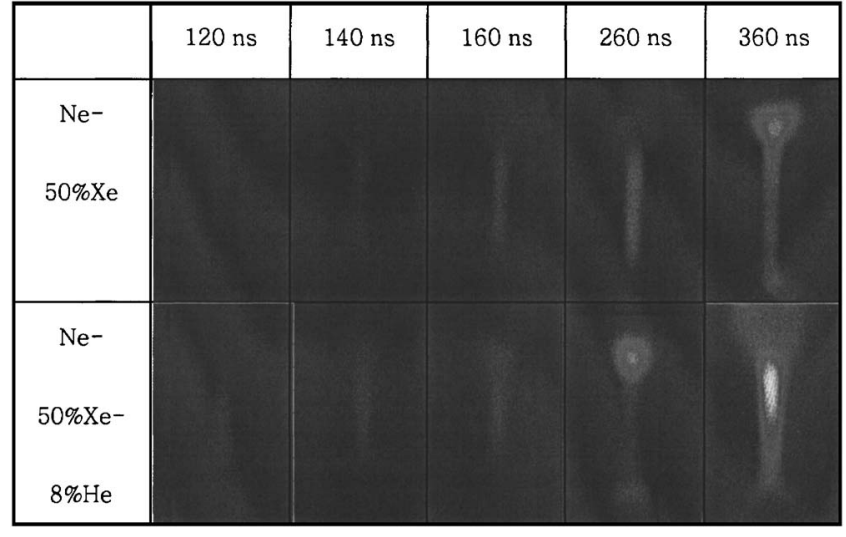

FIG. 6. Variation in ICCD images over time with ac rectangular pulse of $20 \mathrm{kHz}, 5 \mu$ s duty cycle, and $1.6 \mathrm{kV}$.

edges. As such, the first peak component of the $\mathrm{Ne}-50 \% \mathrm{Xe}$ rising edge is sharper than that of the $\mathrm{Ne}-50 \% \mathrm{Xe}-8 \% \mathrm{He}$ rising edge.

In order to assess the initial discharge characteristics, image were taken using an ICCD camera (ICCD4710, Andor) with a $20 \mathrm{~ns}$ gate mode and driving conditions of $20 \mathrm{kHz}$ and $5 \mu$ s duty cycles. Figure 6 shows both Ne-50\% $\mathrm{Xe}$ and $\mathrm{Ne}-50 \% \mathrm{Xe}-8 \% \mathrm{He}$ discharge images within a range of $120-360 \mathrm{~ns}$. In the figure, the top sections show the position of the cathode ( $X$ electrode), while the bottom sections show the location of the anode ( $Y$ electrode). During the initial stage from 120 to $160 \mathrm{~ns}$, the start of the discharge was confirmed. However, the discharge was very cloudy and had a localized band structure. At $260 \mathrm{~ns}, \mathrm{Ne}-50 \% \mathrm{Xe}-8 \%$ $\mathrm{He}$ and $\mathrm{Ne}-50 \%$ Xe both showed distinctive strong regions of discharge in the cathode area. In the case of Ne-50\% $\mathrm{Xe}-8 \% \mathrm{He}$, a stronger round-shaped discharge was seen, yet still confined to a small area. The intensity of the discharge was much larger in $\mathrm{Ne}-50 \%$ Xe- $8 \%$ He compared to $\mathrm{Ne}-$ $50 \% \mathrm{Xe}$. This was also found in the IR emission spectrum results, as shown in Fig. 5. At 360 ns, both cases exhibited broader discharge characteristics across the cathode and anode regions. The discharge characteristics of $\mathrm{Ne}-50 \%$ $\mathrm{Xe}-8 \% \mathrm{He}$ showed a broader distribution, and the highest intensity region was more centered inside the cell compared to the case of $\mathrm{Ne}-50 \% \mathrm{Xe}$. Furthermore, the addition of $\mathrm{He}$ accelerated the dispersion of the discharge in the $\mathrm{Ne}-\mathrm{Xe}$ gas system. Yoon et $\mathrm{al}^{7}{ }^{7}$ previously reported that the peak emission delay in $\mathrm{Ne}-\mathrm{Xe}$, in contrast to a $\mathrm{Ne}-\mathrm{He}$ gas system, originates from the slow formation and movement of the cathode fall head in Ne-Xe. Generally, the discharge breakdown characteristic time for the glow discharge is given by the following equation: ${ }^{8}$

$$
1 / \tau=v\left[\alpha-(1 / d) \ln \left(1+\gamma_{i}\right) / \gamma_{i}\right],
$$

where $v=v_{i} v_{e} /\left(v_{i}+v_{e}\right)$. Here, $v_{i}$ and $v_{e}$ are the velocities of the ions and electrons, respectively, $\alpha$ is the ionization rate by the electrons, $d$ is the discharge distance, and $\gamma_{i}$ is the secondary electron emission coefficient of the ions. The ion velocity is much slower than the electron velocity and $\gamma_{i}$ is negligible for Xe ions. Thus, it is apparent that $\tau_{i}$ is inversely proportional to $v_{i} \alpha$. As the order of magnitude $\alpha$ is almost

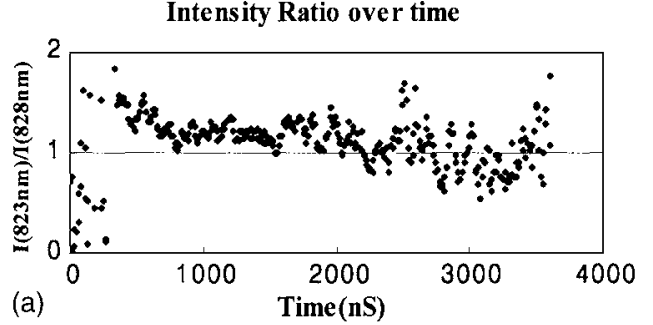

Intensity Ratio over time

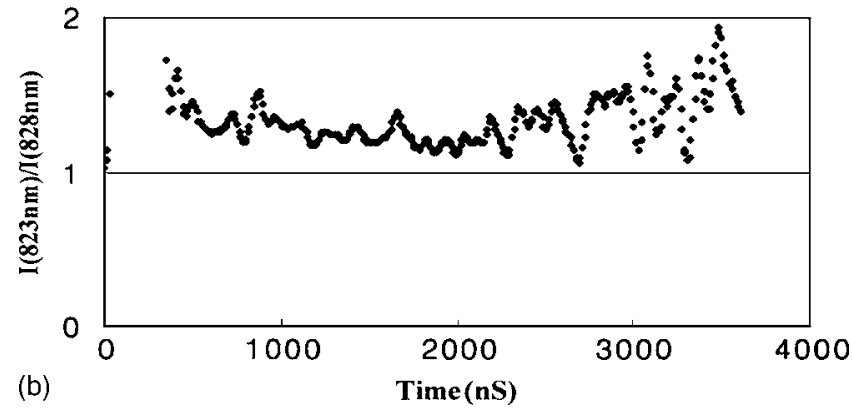

FIG. 7. Intensity ratio of $I_{823 \mathrm{~nm}} / I_{828 \mathrm{~nm}}$ over time: (a) $\mathrm{Ne}-50 \% \mathrm{Xe}$ and (b) $\mathrm{Ne}-50 \% \mathrm{Xe}-8 \% \mathrm{He}$

the same for $\mathrm{Ne}-\mathrm{Xe}$ and $\mathrm{He}-\mathrm{Xe}$, the emission peak time can be principally determined by the xenon-ion velocity. Chanin and Biondi reported that xenon mobility is greater in helium than in neon due to the difference in the scattering cross sections. ${ }^{9}$ At 300 Torr, the xenon-ion mobility is $48.1 \mathrm{~cm}^{2} / \mathrm{V} \mathrm{S}$ in helium and $16.5 \mathrm{~cm}^{2} / \mathrm{V} \mathrm{S}$ in neon. Accordingly, based on the ion mobility and electrical field strength, at the same applied voltage and discharge path, the delayed emission peak appearance depends on the xenon-ion mobility. As seen in Fig. 6, the faster emission peak appearance in the $\mathrm{Ne}-50 \% \mathrm{Xe}-8 \% \mathrm{He}$ gas discharge can partially be explained by this model. When compared to $\mathrm{Ne}-50 \% \mathrm{Xe}$, some of the $\mathrm{Xe}$ ions in the $\mathrm{Ne}-50 \% \mathrm{Xe}-8 \% \mathrm{He}$ gas have higher mobility, which accelerates the appearance of the peak emission.

Figure 7 shows the intensity ratio of $I_{823 \mathrm{~nm}} / I_{828} \mathrm{~nm}$ over time in both $\mathrm{Ne}-50 \% \mathrm{Xe}-8 \% \mathrm{He}$ and $\mathrm{Ne}-50 \% \mathrm{Xe}-8 \% \mathrm{He}$, as derived from Fig. 5. The $823 \mathrm{~nm}$ photon is emitted during the transition from the ${ }^{2} P_{6}$ excited state to the ${ }^{3} P_{2}$ metastable state of Xe. The $828 \mathrm{~nm}$ photon is emitted during the transition from the ${ }^{2} P_{5}$ excited state to the ${ }^{3} P_{1}$ excited state of Xe. The $\mathrm{Hg}$-free lamp containing Xe utilizes $147 \mathrm{~nm}$ photons emitted from the ${ }^{3} P_{1}$ excited state of the Xe monomer and $173 \mathrm{~nm}$ photons emitted from the ${ }^{3} P_{2}$ state of the Xe dimer. IR intensities of 823 and $828 \mathrm{~nm}$ are proportional to the vuv intensity, which is related to the luminance and efficacy of the lamp. The integrated intensity ratio was $\sim 8 \%$ higher in the $\mathrm{Ne}-50 \% \mathrm{Xe}-8 \% \mathrm{He}$, as determined from Fig. 5, while the IR emission from $828 \mathrm{~nm}$ was dominant inside the discharge cell, as illustrated in Fig. 7. Generally, with a Xe discharge, the vuv radiation mainly consists of the Xe atomic resonance at $147 \mathrm{~nm}$ and $\mathrm{Xe}^{*}\left({ }^{3} P_{2}\right)$ emission at $173 \mathrm{~nm} .{ }^{10}$ For the Xe partial pressure and lamp dimensions ${ }^{11,12}$ generally used in Xe backlights and the flat lamp in this paper, more than $90 \%$ of the radiation is $\mathrm{Xe}^{*}\left({ }^{3} P_{2}\right)$ emission at $173 \mathrm{~nm}$, which is derived from $\mathrm{Xe}^{* * *}$ and infrared $823 \mathrm{~nm}$. As 
such, a higher-intensity level of infrared $823 \mathrm{~nm}$ indicates more emission at $173 \mathrm{~nm}$. Therefore, the reason for the higher luminance and efficacy in $\mathrm{Ne}-50 \% \mathrm{Xe}-8 \% \mathrm{He}$ was due to the higher infrared $823 \mathrm{~nm}$ emission intensity, indicating the existence of $\mathrm{Xe}^{*}\left({ }^{3} P_{2}\right)$.

\section{SUMMARY}

An experimental $\mathrm{Hg}$-free flat lamp was developed based on a dual-coplanar multielectrode fluorescent lamp. The discharge characteristics of the flat lamp were investigated using brightness-efficiency measurements and the IR spectrum and ICCD characteristics. The level of brightness was above $14900 \mathrm{~cd} / \mathrm{m}^{2}$ under the conditions of $\mathrm{Ne}-50 \% \mathrm{Xe}-8 \% \mathrm{He}$ gas composition, 150 Torr pressure, and $20 \mathrm{kHz}$ ac pulse. The ICCD results revealed faster and wider spread discharge in the $\mathrm{Ne}-50 \% \mathrm{Xe}-8 \% \mathrm{He}$ gas composition. The effect of adding $\mathrm{He}$ to $\mathrm{Ne}-50 \% \mathrm{Xe}$, as confirmed by ICCD images, revealed a faster peak emission. This implies the existence of a certain portion of $\mathrm{Xe}$ ions with a higher mobility in $\mathrm{Ne}-$ $50 \% \mathrm{Xe}-8 \% \mathrm{He}$ gas. From the gated IR emission spectrum, the integrated intensity ratio of $I_{823 \mathrm{~nm}} / I_{828 \mathrm{~nm}}$ was $\sim 8 \%$ higher in $\mathrm{Ne}-50 \% \mathrm{Xe}-8 \%$ He than in $\mathrm{Ne}-50 \%$ Xe under the same pressure and applied voltage conditions.

${ }^{1}$ Y. Ikeda, T. Shiga, and S. Mikoshiba, SID Int. Symp. Digest Tech. Papers 2000, 938.

${ }^{2}$ M. Ilmer, R. Lecheler, H. Schweizer, and M. Seibold, SID Int. Symp. Digest Tech. Papers 2000, 931.

${ }^{3}$ H. B. Park, K. Y. Kim, and J. H. Hong, SID Int. Symp. Digest Tech. Papers 2003, 1138

${ }^{4}$ G. Veronis, U. S. Ian, and V. P. Pasko, IEEE Trans. Plasma Sci. 28, 1276 (2000).

${ }^{5}$ D.-Z. Wang, Y.-H. Wang, Y.-T. Zhang, B. Qi, and C.-S. Ren, Abstracts of the Seventh Asian Pacific Conference on Plasma Science and Technology, Fukuoka, 2004 (unpublished), p. 51.

${ }^{6}$ K. C. Choi, B.-J. Rhee, and H.-N. Lee, IEEE Trans. Plasma Sci. 31, 329 (2003).

${ }^{7}$ C. K. Yoon, J. H. Seo, and K. W. Whang, IEEE Trans. Plasma Sci. 28 , 1029 (2000)

${ }^{8}$ E. Nasser, Fundamentals of Gaseous Ionization and Plasma Electronics (Wiley, New York, 1970).

${ }^{9}$ L. M. Chanin and M. A. Biondi, Phys. Rev. 107, 1219 (1957).

${ }^{10}$ R. T. Mcgrath, R. Veerasingam, J. A. Hunter, P. D. Rockett, and R. B. Campbell, IEEE Trans. Plasma Sci. 26, 1532 (1998).

${ }^{11}$ T. Holstein, Phys. Rev. 83, 1159 (1951).

${ }^{12}$ J. Meunier, Ph. Belenguer, and J. P. Boeuf, J. Appl. Phys. 78, 731 (1995). 